\title{
A PERFECTLY NORMAL, LOCALLY COMPACT, NONCOLLECTIONWISE NORMAL SPACE FROM $\diamond^{*}$
}

\author{
PEG DANIELS AND GARY GRUENHAGE ${ }^{1}$
}

\begin{abstract}
A perfectly normal, locally compact, collectionwise- $T_{2}$, noncollectionwise normal space is constructed using $\searrow^{*}$, a combinatorial axiom which holds in Gödel's constructible universe $L$. The construction answers questions of F. Tall and S. Watson.
\end{abstract}

1. Introduction. The existence of spaces which are perfectly normal and locally compact, but not collectionwise normal (CWN) is known to be consistent with, and independent of, the usual axioms of set theory. Under Martin's Axiom plus the negation of the Continuum Hypothesis $(\mathrm{CH})$ such spaces exist-an $\omega_{1^{-}}$ Cantor tree, for example. On the other hand, F. Tall $[\mathbf{T}]$ obtained a model of set theory in which all perfectly normal, locally compact spaces are $\mathrm{CWN},{ }^{2}$ by adjoining $\aleph_{2}$ random reals to a model of $\mathrm{V}=\mathrm{L}$. He asked whether this result follows from $\mathrm{V}=\mathrm{L}$ alone. S. Watson $[\mathbf{W}]$ showed that in $\mathrm{L}$ all normal, locally compact spaces are collectionwise Hausdorff $(\mathrm{CWH})$; in fact, that they are collectionwise normal with respect to compact sets. Since all known examples of (perfectly) normal, locally compact, non-CWN spaces were in fact non-CWH, he was led to ask whether all (perfectly) normal, locally compact, CWH spaces are CWN. In this paper we answer the questions of Tall and Watson in the negative by constructing, assuming $\diamond^{*}$ (which holds in L), a perfectly normal, locally compact, $\mathrm{CWH}$, non-CWN space.

2. Construction of the example. Our example is obtained by modifying the technique of constructing the so-called "Kunen line", as described in [JKR]. This technique is a machine which works uner $\mathrm{CH}$ : If the input is a space $(X, \tau)$, where $X$ has cardinality $\aleph_{1}$ and $\tau$ is a first countable, Hausdorff topology, the output is $\left(X, \tau^{\prime}\right)$, where $\tau^{\prime}$ is finer than $\tau$, and is locally compact, locally countable, regular, and not Lindelöf. If, in addition, $\tau$ is hereditarily separable and perfectly normal, then so is $\tau^{\prime}$ (hereditary separability of the input is used to show the normality of the output), and thus $\tau^{\prime}$ will be CWN as well. In [JKR] the machine is applied to $\mathbf{R}$ to yield a perfectly normal, locally compact, hereditarily separable, non-Lindelöf space.

Our modification of this machine does not require a hereditarily separable input to get a normal output. Instead, we need the input $(X, \tau)$ to be a first countable,

Received by the editors July 13,1984 and, in revised form, November 27,1984 . Presented at the Annual Spring Topology Conference, held at Auburn University, March, 1984.

1980 Mathematics Subject Classification. Primary 54D15.

${ }^{1}$ Research supported by NSF Grant MCS-8301932.

${ }^{2}$ Nyikos $[\mathbf{N}]$ showed earlier that this holds under the axiom PMEA, but large cardinals are needed to prove the consistency of PMEA. 
perfectly normal CWH space of cardinality $\aleph_{1}$, and we need left-separated ${ }^{3}$ subsets of $X$ to be $\sigma$-closed discrete (all Moore spaces, for example, satisfy the latter condition). Given such an input space, the output will be locally countable, locally compact, perfectly normal, $\mathrm{CWH}$, and non-Lindelöf. If the input is also non-CWN, so is the output. Thus, applying our machine to Fleissner's $\mathrm{CH}$ example $[\mathbf{F}]$ of a normal, CWH, nonmetrizable Moore space yields our desired example.

In order to work, our modification requires the following axiom, $\diamond^{*}$, a strengthening of $\diamond$ which holds in $L$ :

$\diamond^{*}$ : There exists a sequence $\left\langle A_{\alpha}\right\rangle_{\alpha<\omega_{1}}$ such that

(i) $A_{\alpha} \subset P(\alpha)$ and $\left|A_{\alpha}\right| \leq \omega$ for all $\alpha<\omega_{1}$, and

(ii) for each $A \subset \omega_{1},\left\{\alpha: A \cap \alpha \in A_{\alpha}\right\}$ contains a closed unbounded subset of $\omega_{1}$.

A sequence $\left\langle A_{\alpha}\right\rangle_{\alpha<\omega_{1}}$ that satisfies (i) and (ii) is called a $\diamond^{*}$-sequence.

$\diamond^{*}$ seems to be necessary to show that, given an input $(X, \tau)$ and a subset $A$ of $X$ closed in the output $\left(X, \tau^{\prime}\right), \operatorname{cl}_{\tau}(A)-A$ is $\sigma$-left-separated in $(X, \tau)$, hence $\sigma$-closed discrete in $(X, \tau)$ by an assumption on the input space. It is then fairly easy to see that the other assumptions on the input space imply the claimed properties of the output. (By way of comparison, the key fact in the [JKR] technique is that $\mathrm{cl}_{\tau}(A)-A$ is countable.)

THEOREM $\left(\diamond^{*}\right)$. Suppose $|X|=\aleph_{1}$, and $\tau$ is a first countable, ${ }^{4}$ perfectly normal, $C W H$ topology on $X$ such that left-separated subsets of $X$ are $\tau$-closed discrete. Then there exists a finer topology $\tau^{\prime}$ on $X$ such that $\left(X, \tau^{\prime}\right)$ is locally countable, locally compact, perfectly normal, and $C W H$. If $(X, \tau)$ is not $C W N$, then neither is $\left(X, \tau^{\prime}\right)$.

Proof. Assume the hypothesis of the theorem. Since $|X|=\aleph_{1}$, let us identify $X$ with $\omega_{1}$. For each $\alpha \leq \omega_{1}$, let $X_{\alpha}=\alpha$. Let $\left\langle A_{\alpha}: \alpha<\omega_{1}\right\rangle$ be a $\diamond^{*}$-sequence. We construct, by induction, a Hausdorff topology $\tau_{\alpha}$ on $X_{\alpha}$ so that, if $\beta<\alpha \leq \omega_{1}$, the following hold:

(1) $\tau_{\beta}=\tau_{\alpha} \cap P\left(X_{\beta}\right)$ (this condition implies that $\tau_{\beta}$ is the relative topology on $X_{\beta}$ in the space $\left.\left(X_{\alpha}, \tau_{\alpha}\right)\right)$,

(2) $\tau_{\alpha}$ is first countable and locally compact,

(3) $\tau_{\alpha}$ is finer than the original subspace topology on $X_{\alpha}$,

(4) if $\delta \leq \beta, A \in A_{\delta}$, and $\beta \in \operatorname{cl}_{\tau}(A)$, then $\beta \in \operatorname{cl}_{\tau_{\alpha}}(A)$, and

(5) $\tau_{\alpha}$ is regular, zero-dimensional, and, if $\alpha<\omega_{1}$, metrizable.

For $\alpha \leq \omega$, let $\tau_{\alpha}$ be the discrete topology on $X_{\alpha}$. Suppose we have defined $\tau_{\beta}$ for each $\beta<\alpha$, for some $\alpha \leq \omega_{1}$, so that (1)-(5) hold.

If $\alpha$ is a limit, let $\tau_{\alpha}$ be the topology generated by $\bigcup_{\beta<\alpha} \tau_{\alpha}$, i.e.

$$
\tau_{\alpha}=\left\{U \subset X_{\alpha}: U \cap X_{\beta} \in \tau_{\beta} \text { for each } \beta<\alpha\right\} .
$$

It is not difficult to check that (1)-(5) hold in this case.

If $\alpha$ is a successor, say $\alpha=\alpha^{\prime}+1$, proceed as follows. If there is no $A \in \bigcup_{\delta<\alpha^{\prime}} A_{\delta}$ such that $\alpha^{\prime} \in \operatorname{cl}_{\tau}(A)$, let $\alpha^{\prime}$ be isolated, and let $\tau_{\alpha}$ be generated by $\tau_{\alpha^{\prime}} \cup\left\{\left\{\alpha^{\prime}\right\}\right\}$. If there is such an $A$, let $\left\{B_{n}: n \in \omega\right\}$ list all such, with each occurring infinitely many times in the listing. Since $\tau$ is first countable, let $\left\{V_{n}\left(\alpha^{\prime}\right): n \in \omega\right\}$ be a decreasing

\footnotetext{
${ }^{3} \mathrm{~A}$ space $X$ is left-separated if there is a well-ordering $<$ of $X$ such that, for each $x \in X$, $x \notin\{y: y<x\}^{-}$.

4 "First countable" may be replaced in this theorem by the following weaker condition: whenever $x \in X$ and $x \in \bigcap_{n \in \omega} \overline{B_{n}}$, there exist $x_{n} \in B_{n}$ with $x_{n} \rightarrow x$.
} 
base at $\alpha^{\prime}$, and choose $p_{n}$ for each $n$ such that $p_{n} \in B_{n} \cap V_{n}\left(\alpha^{\prime}\right)$. Since $\left\{p_{n}: n \in \omega\right\}$ is closed discrete in the original subspace topology on $X_{\alpha^{\prime}}$, it is closed discrete in $\tau_{\alpha^{\prime}}$. By assumption, $\tau_{\alpha^{\prime}}$ is zero-dimensional, locally compact, and metrizable (and therefore normal and $\mathrm{CWH}$ ), so for each $n \in \omega$ we may let $K_{n}$ be a clopen compact set in $\tau_{\alpha^{\prime}}$ containing $p_{n}$ such that $K_{n} \subset V_{n}\left(\alpha^{\prime}\right) \cap X_{\alpha^{\prime}}$ and $\left\{K_{n}: n \in \omega\right\}$ is discrete. For each $n \in \omega$, let $U_{n}\left(\alpha^{\prime}\right)=\left\{\alpha^{\prime}\right\} \cup \bigcup\left\{K_{m}: m \geq n\right\}$, and let $\tau_{\alpha}$ be the topology generated by $\tau_{\alpha^{\prime}} \cup\left\{U_{n}\left(\alpha^{\prime}\right): n \in \omega\right\}$. In either of these cases it is easy to check that (1)-(5) hold.

Let us denote the topology $\tau_{\omega_{1}}$ on $X$ by $\tau^{\prime}$. Clearly, $\left(X, \tau^{\prime}\right)$ is locally countable and locally compact. We wish to show it is also perfectly normal and CWH. With this in mind, we now prove the key fact: If $A$ is closed in $\left(X, \tau^{\prime}\right)$, then $\operatorname{cl}_{\tau}(A) \backslash A$ is $\sigma$-closed discrete in $(X, \tau)$. Suppose $A$ is closed in $\left(X, \tau^{\prime}\right)$, and let $A^{*}=\operatorname{cl}_{\tau}(A) \backslash A$. Since $\tau$ is first countable, for each $\beta \in A^{*}$ we may let $\phi(\beta)<\omega_{1}$ such that $\beta \in$ $\operatorname{cl}_{\tau}(A \cap \phi(\beta))$. Let $C=\left\{\alpha<\omega_{1}: \beta<\alpha\right.$ implies $\left.\phi(\beta)<\alpha\right\}$. $C$ is a closed unbounded subset of $\omega_{1}$. By $\diamond^{*}$, let $C^{\prime}$ be a closed unbounded subset of $\omega_{1}$ such that, for each $\alpha \in C^{\prime}, A \cap \alpha \in A_{\alpha}$. Let $D=C \cap C^{\prime}$, and let $\left\{d_{\alpha}: \alpha<\omega_{1}\right\}$ be an increasing enumeration of $D$. Without loss of generality we may assume $d_{0}=0$. Then $\omega_{1}=\bigcup_{\alpha<\omega_{1}}\left[d_{\alpha}, d_{\alpha+1}\right)$. For each $\alpha<\omega_{1}$, enumerate the countable set $A^{*} \cap\left[d_{\alpha}, d_{\alpha+1}\right)$ by $\left\{b_{\alpha n}: n \in \omega\right\}$. For each $n \in \omega$, let $B_{n}=\left\{b_{\alpha n}: \alpha<\omega_{1}\right\}$. Each $B_{n}$ is left-separated in $\tau$ : If not, there is an $\alpha<\omega_{1}$ such that $b_{\alpha n} \in \operatorname{cl}_{\tau}\left(\left\{b_{\beta n}: \beta<\alpha\right\}\right)$, which implies that $b_{\alpha n} \in \operatorname{cl}_{\tau}\left(A^{*} \cap d_{\alpha}\right)$ : it is easy to check that this implies that $b_{\alpha n} \in \operatorname{cl}_{\tau}\left(A \cap d_{\alpha}\right)$. Since $d_{\alpha} \leq b_{\alpha n}$ and $A \cap d_{\alpha} \in A_{d_{\alpha}}$, this means that $b_{\alpha n} \in$ $\operatorname{cl}_{\tau^{\prime}}\left(A \cap d_{\alpha}\right)$, so $b_{\alpha n} \in A$, which is a contradiction. Since $B_{n}$ is left-separated in $(X, \tau)$, we have by our assumptions on $(X, \tau)$ that $B_{n}$ is $\sigma$-closed discrete in $(X, \tau)$. Hence $A^{*}$, which is the union of the $B_{n}$ 's, is $\sigma$-closed discrete in $(X, \tau)$.

We now show $\left(X, \tau^{\prime}\right)$ is perfectly normal. Equivalently, we show that if $A$ is closed in $\left(X, \tau^{\prime}\right)$, then there is a sequence of sets $\left\langle V_{n}: n \in \omega\right\rangle$ such that each $V_{n} \in \tau^{\prime}$ and $A=\bigcap_{n \in \omega} V_{n}=\bigcap_{n \in \omega} \operatorname{cl}_{\tau^{\prime}}\left(V_{n}\right)$. Since $(X, \tau)$ perfectly normal, let $U_{n} \in \tau$ for each $n$ such that $\operatorname{cl}_{\tau}(A)=\bigcap_{n \in \omega} U_{n}=\bigcap_{n \in \omega} \operatorname{cl}_{\tau}\left(U_{n}\right)$. Let $\operatorname{cl}_{\tau}(A) \backslash A=\bigcup_{n \in \omega} B_{n}$, where each $B_{n}$ is closed discrete in $\tau$. Since $\tau$ is CWH and normal, let $\left\{V_{b n}: b \in B_{n}\right\}$ be a discrete collection of open sets in $\tau$ separating the point in $B_{n}$. Since $\tau^{\prime}$ is finer than $\tau$ and is zero-dimensional, for each $n \in \omega$ and each $b \in B_{n}$, let $W_{b n}$ be clopen in $\tau^{\prime}$ such that $b \in W_{b n} \subset V_{b n} \cap(X \backslash A)$; since $\left\{W_{b n}: b \in B_{n}\right\}$ is discrete in $\tau^{\prime}, \bigcup_{b \in B_{n}} W_{b n}$ is clopen in $\tau^{\prime}$, and it is easy to check that

$$
A=\bigcap_{n \in \omega} U_{n} \cap \bigcap_{n \in \omega}\left(X \backslash \bigcup_{b \in B_{n}} W_{b n}\right)=\bigcap_{n \in \omega} \operatorname{cl}_{\tau^{\prime}}\left(U_{n}\right) \cap \bigcap_{n \in \omega}\left(X \backslash \bigcup_{b \in B_{n}} W_{b n}\right) .
$$

Shelah $[\mathbf{S}]$ has shown that $\diamond^{*}$ implies that every normal space of character $\leq \aleph_{1}$ is $\aleph_{1}$-CWH (every closed discrete set of size $\omega_{1}$ can be separated), so our space $\left(X, \tau^{\prime}\right)$ is CWH. This can also be proved directly in a manner similar to the proof of the key fact above, by showing that a closed discrete set in $\left(X, \tau^{\prime}\right)$ is $\sigma$-left-separated, and hence $\sigma$-closed discrete, in $(X, \tau)$. We omit this proof.

Finally, let us assume that $(X, \tau)$ is not CWN, and show that neither is $\left(X, \tau^{\prime}\right)$. Let $H=\left\{H_{\alpha}: \alpha<\omega_{1}\right\}$ be a discrete collection of closed sets that cannot be separated in $(X, \tau) . H$ is also a discrete collection of closed sets in $\left(X, \tau^{\prime}\right)$. Suppose $H$ can be separated in $\left(X, \tau^{\prime}\right)$. Let $U_{\alpha} \in \tau^{\prime}$ for each $\alpha<\omega_{1}$ such that $H_{\alpha} \subset U_{\alpha}$ and $\left\{U_{\alpha}: \alpha<\omega_{1}\right\}$ is pairwise disjoint. We will show that we can get a separation 
of $H$ in $(X, \tau)$. It is sufficient to show the following:

(*) For each $\alpha<\omega_{1}$ there is a collection $\left\{V_{\alpha n}: n \in \omega\right\} \subset \tau$ such that $H_{\alpha} \subset$ $\bigcup_{n \in \omega} V_{\alpha n},\left\{V_{\alpha n}: \alpha<\omega_{1}\right\}$ is discrete in $(X, \tau)$ for each $n \in \omega$, and if $\alpha \neq \beta$ in $\omega_{1}$, $\operatorname{cl}_{\tau}\left(V_{\alpha n}\right) \cap H_{\beta}=\varnothing$.

Since a closed set in $\left(X, \tau^{\prime}\right)$ is equal to a closed set in $(X, \tau)$ minus a $\sigma$-closed discrete (in $(X, \tau))$ set, an open set in $\left(X, \tau^{\prime}\right)$ is equal to an open set in $(X, \tau)$ plus a $\sigma$-closed discrete (in $(X, \tau))$ set. So for each $\alpha<\omega_{1}$, let $U_{\alpha}=U_{\alpha}^{\prime} \cup \bigcup_{n \in \omega} B_{\alpha n}$, where $U_{\alpha}^{\prime} \in \tau$ and each $B_{\alpha n}$ is closed discrete in $(X, \tau)$. We first consider the points in $H_{\alpha} \backslash U_{\alpha}^{\prime}$. For each $\alpha<\omega_{1}$ and each $n \in \omega$, let $H_{\alpha n}=\left(H_{\alpha} \backslash U_{\alpha}^{\prime}\right) \cap B_{\alpha n}$. Since $\left\{H_{\alpha n}: \alpha<\omega_{1}\right\}$ is a discrete collection of closed sets in $(X, \tau)$ and each $H_{\alpha n}$ is closed discrete in $(X, \tau), \bigcup_{\alpha<\omega_{1}} H_{\alpha n}$ is closed discrete in $(X, \tau)$ for each $n \in \omega$. Since $\tau$ is normal and CWH, let $\left\{V_{b n}: b \in \bigcup_{\alpha<\omega_{1}} H_{\alpha n}\right\}$ be a discrete collection of open sets in $(X, \tau)$ separating $\bigcup_{\alpha<\omega_{1}} H_{\alpha n}$. Then $\left\{\bigcup\left\{V_{b n}: b \in H_{\alpha n}\right\}: \alpha<\omega_{1}\right\}$ is a discrete collection of open sets in $(X, \tau)$ separating $\left\{H_{\alpha n}: \alpha<\omega_{1}\right\}$. Since $\tau$ is normal, we may assume without loss of generality that, if $\alpha \neq \beta$ in $\omega_{1}$,

$$
\operatorname{cl}_{\tau}\left(\bigcup\left\{V_{b n}: b \in H_{\alpha n}\right\}\right) \cap H_{\beta}=\varnothing .
$$

Now let us consider the points of $H_{\alpha} \cap U_{\alpha}^{\prime}$. Since $\tau$ is perfectly normal, we may let $U_{\alpha}^{\prime}=\bigcup_{n \in \omega} K_{\alpha n}$ so that each $K_{\alpha n}$ is closed. Then $\left\{H_{\alpha} \cap K_{\alpha n}: \alpha<\omega_{1}\right\}$ is a discrete collection of closed sets in $(X, \tau)$ that is separated by $\left\{U_{\alpha}^{\prime}: \alpha<\omega_{1}\right\}$. By normality we may therefore separate each $\left\{H_{\alpha} \cap K_{\alpha n}: \alpha<\omega_{1}\right\}$ by an appropriate discrete collection of open sets in $(X, \tau)$. Thus we have satisfied $(*)$, and so $H$ can be separated in $(X, \tau)$, contradicting our assumption. Therefore, $H$ cannot be separated in $\left(X, \tau^{\prime}\right)$, and so $\left(X, \tau^{\prime}\right)$ is not CWN.

COROLlaRY $\left(\diamond^{*}\right)$. There exists a perfectly normal, locally compact, $C W H$, non-CWN space.

Proof. Apply the theorem to Fleissner's CH example $[\mathbf{F}]$ of a (perfectly) normal nonmetrizable Moore space.

\section{REFERENCES}

[F] W. G. Fleissner, Normal nonmetrizable Moore spaces from continuum hypothesis or nonexistence of inner models with measurable cardinals, Proc. Nat. Acad. Sci. U.S.A. 79 (1982), 1371-1372.

[JKR] I. Juhasz, K. Kunen and M. E. Rudin, Two more hereditarily separable, non-Lindelöf spaces, Canad. J. Math. 28 (1976), 998-1005.

[N] P. J. Nyikos, A provisional solution to the normal Moore space problem, Proc. Amer. Math. Soc. 78 (1980), 429-435.

[S] S. Shelah, $A$ note in general topology: if $\diamond_{\aleph_{1}}^{*}$ then any normal space of character $\leq \aleph_{1}$ is $\aleph_{1}-\mathrm{CWH}$, preprints in Math. Logic (1979).

[T] F. D. Tall, Collectionwise normality without large cardinals, Proc. Amer. Math. Soc. 85 (1982), $100-102$.

[W] W. S. Watson, Locally compact normal spaces in the constructible inverse, Canad. J. Math. 34 (1982), 1091-1096.

Department of MATHEMATICS, University of TORONTO, TORONTO, OntARio M5S 1A1, CANADA

Department of Mathematics, Auburn University, Auburn, Alabama 36849 (Current address of both authors) 